\title{
Serons-nous soignés contre notre volonté?
}

\section{Hans Stalder}

Dr med., membre de la rédaction

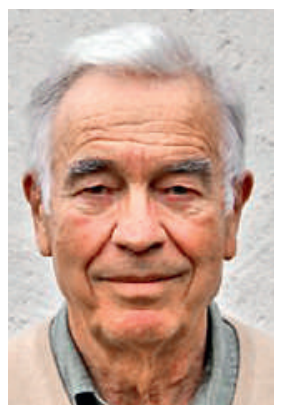

Souvent les personnes âgées expriment le désir de vouloir plutôt mourir que souffrir de démence. Dans une étude anglaise presque 60\% des personnes interrogées [1] ont même exprimé le vœu d'accéder à l'euthanasie active en cas de démence et en Belgique plus que $71 \%$ se sont exprimées dans ce sens [2]. Pourquoi ce vœu ne peut-il être exaucé? L'autonomie est sauvegardée, car il s'agit d'un désir exprimé volontairement; il n'y a pas de malfaisance concernant la personne impliquée et il n'en résulte aucune charge pour la société. Au contraire, car la personne impliquée exprime souvent le souhait de ne pas être un fardeau pour ses proches et pour les soignants.

Une telle possibilité est exclue en Suisse car l'euthanasie active est considérée comme un homicide, punissable même s'il y a une demande de la personne concernée et un mobile honorable, notamment la pitié (CP art. 114). Pour un suicide assisté, qui est poursuivi chez nous seulement s'il y a un mobile égoïste, il faut que le patient soit capable de discernement, ce qui n'est évidemment pas assuré en cas de démence avérée. C'est pour cette raison qu'Exit suisse-alémanique a clairement décliné cette procédure dans cette situation. On pourrait penser que l'euthanasie serait pratiquée dans un pays comme la Hollande, où depuis 2002 elle est autorisée aussi en cas de démence, pour autant que des directives anticipées aient été écrites dans ce sens (en Belgique une proposition de loi similaire a été déposée en 2015 [2]). Le fait est que parmi les 2200 personnes qui ont explicitement demandé dans leurs directives anticipées l'euthanasie en cas de démence, aucune n'a obtenu sa réalisation [3]. Il y a deux raisons pour l'expliquer: dans la loi hollandaise sur l'euthanasie il est entre autres exigé qu'il y ait "souffrance sans espoir et insupportable». Chez un patient dément, la souffrance, évaluée par les soignants, peut paraître insupportable, mais être certain qu'elle l'est vraiment aussi pour le patient est impossible à savoir sans un véritable dialogue. Et même si les soignants ou les proches en sont persuadés, la loi stipule que «le patient et le médecin doivent être convaincus qu'il n'y a pas d'autres perspectives». Comment être sûr que c'est le cas? Peut-être le patient devenu dément a-t-il changé d'avis sans pouvoir l'exprimer? C'est en raison de ce manque de communication - élément indispensable dans cette situation délicate - entre le patient et le médecin que même les médecins favorables à l'euthanasie en d'autres circonstances l'ont toujours refusée chez des patients déments [4].

On doit conclure que malgré le fait qu'une majorité de personnes aimeraient pouvoir mourir si elles souffrent de démence, il s'avère impossible de satisfaire cette demande. Si l'on transpose les données d'Angleterre et de Belgique $[1,2]$ en Suisse, entre $60 \%$ et $70 \%$ des quelque 120000 patients atteints de démence, soit plus de 70000 , auraient préféré mourir plutôt qu'être soignés...

Il ne reste aux personnes pour qui cette situation est insupportable que le choix de mourir par un suicide assisté entre l'annonce du diagnostic de démence débutante, le minimal cognitive impairment (MCI), quand elles sont encore capables de discernement, et le moment où elles sombrent dans la démence. Mais il faut savoir que dans certaines études [5] la probabilité de devenir dément en cas de MCI peut être aussi bas que $5 \%$ par an (en moyenne environ 10\%) et qu'une partie des patients avec ce diagnostic récupéreront leur mémoire. Veut-on vraiment mourir précocement quand on a une chance non négligeable de vivre encore un bon moment sans devenir dément?

La situation me semble inextricable, mais peut-être, chers lecteurs, avez-vous une solution?

\section{Références}

1 Williams N, Dunford C, Knowles A, Warner J. Public attitudes to life-sustaining treatments and euthanasia in dementia. Int J Geriatr Psychiatry. 2007;22:1229-34.

2 Chambre des représentants de Belgique. Proposition de loi 1013/001. https://www.lachambre.be/

3 Rurup ML, Onwuteaka-Philipsen BD, van der Heide A, van der Wal G, van der Maas PJ. Physicians' experiences with demented patients with advance euthanasia directives in the Netherlands. J Am Geriatr Soc. 2005;53:1138-44.

4 Bolt EE, Snijdewind MC, Willems DL, van der Heide A, Onwuteaka-Philipsen BD. Can physicians conceive of performing euthanasia in case of psychiatric disease, dementia or being tired of living? J Med Ethics. 2015;41:592-8.

5 Langa KM, Levine DA. The diagnosis and management of mild cognitive impairment: a clinical review. JAMA. 2014;312:2551-61. 